\title{
$\gamma$-secretase inhibitor up-regulates vascular endothelial growth factor receptor-2 and endothelial nitric oxide synthase
}

\author{
YU-HUI ZOU, YI-QUN CAO, LAI-XING WANG, YU-HUI ZHANG, ZHI-JIAN YUE and JIAN-MIN LIU \\ Department of Neurosurgery, Changhai Hospital, Shanghai 200433, P.R. China
}

Received February 4, 2011; Accepted April 11, 2011

DOI: $10.3892 / \mathrm{etm} .2011 .257$

\begin{abstract}
Although previous studies have shown that $\gamma$-secretase inhibitors significantly suppress tumor growth via anti-angiogenesis, the mechanism involved in the regulation of tumor angiogenesis by $\gamma$-secretase inhibitors has not been clearly understood. The objective of this study was to investigate the regulation of vascular endothelial growth factor receptor (VEGFR) and endothelial nitric oxide synthase (eNOS) by a $\gamma$-secretase inhibitor in the H5V mouse microvascular endothelial cell line. H5V cells were cultured with different concentrations of the $\gamma$-secretase inhibitor DAPT for $48 \mathrm{~h}$ and with $100 \mu \mathrm{mol} / 1 \mathrm{DAPT}$ at different incubation times. Protein and mRNA expression of VEGFR-1, VEGFR-2, VEGFR-3 and eNOS was measured by Western blotting and real-time PCR, respectively. The VEGFR-2 kinase inhibitor was used to assess the role of VEGFR-2 in eNOS regulation. We found that the $\gamma$-secretase inhibitor DAPT increased protein and mRNA expression of VEGFR-2 and eNOS, but decreased VEGFR-1 expression and had no significant effect on VEGFR-3. Up-regulation of eNOS was blocked by the VEGFR-2 kinase inhibitor. In conclusion, the $\gamma$-secretase inhibitor enhances VEGFR-2 and eNOS expression, and the up-regulation of eNOS is dependent on an increase in VEGFR-2. Thus, we suggest that administration of the $\gamma$-secretase inhibitor be combined with disruption of eNOS or interruption of VEGF signaling, which may improve the anti-angiogenic efficacy in tumor treatments.
\end{abstract}

\section{Introduction}

In a T-cell acute lymphoblastic leukemia xenograft model, systemic administration of $\gamma$-secretase inhibitor was found to result in significant regression of tumors directly via apoptosis, and indirectly via disturbance of tumor angiogenesis through

Correspondence to: Dr Zhi-Jian Yue or Dr Jian-Min Liu, Department of Neurosurgery, Changhai Hospital, 168 Changhai Road, Shanghai 200433, P.R. China

E-mail: yuezj638@163.com; liu118@vip.163.com

Key words: angiogenesis, vascular endothelial growth factor receptor, endothelial nitric oxide synthase the inhibition of non-cell-autonomous Notch signaling (1). Bone marrow-derived vascular precursor cells pre-treated with the $\gamma$-secretase inhibitor DAPT failed to induce angiogenesis at the wound site and did not promote wound healing in vivo (2). Thus, the $\gamma$-secretase inhibitor may be applied as a pharmacological regulator of angiogenesis. However, the mechanism of angiogenesis regulation by $\gamma$-secretase inhibitors has not been clearly understood.

Vascular endothelial growth factor receptor (VEGFR) is the dominant angiogenic factor, and VEGFR inhibitors are useful in treating cancer via anti-angiogenesis (3). Moreover, nitric oxide (NO), synthesized by endothelial nitric oxide synthase (eNOS), is the most effective endothelium-derived relaxing factor which leads to vasodilation and better microvascular perfusion $(4,5)$. Thus, we used DAPT $\{\mathrm{N}$-[N-(3,5-difluorophenacetyl)-L-alanyl]S-phenylglycine t-butyl ester\}, a $\gamma$-secretase inhibitor, to determine the role of this $\gamma$-secretase inhibitor on VEGFR and eNOS regulation in in vitro models.

\section{Materials and methods}

Cell culture and reagents. The mouse microvascular endothelial H5V cell line was obtained from the Cell Bank of the Shanghai Institute of Biological Sciences, Chinese Academy of Sciences. H5V cells were maintained in DMEM (Gibco) supplemented with $10 \%$ fetal bovine serum (FBS; PAA, Pasching, Austria). The cells were incubated at $37^{\circ} \mathrm{C}$ in a $5 \%$ $\mathrm{CO}_{2}$-humidified atmosphere. DAPT and VEGFR-2 kinase inhibitors were purchased from Sigma Aldrich and Merck, respectively. DAPT was dissolved in DMSO (Sigma Aldrich) to concentrations of $25 \times 10^{3}, 50 \times 10^{3}, 75 \times 10^{3}$ and $100 \times 10^{3} \mu \mathrm{mol} / 1$.

DAPT treatment and VEGFR expression. H5V cells $\left(1 \times 10^{6}\right)$ were cultured with DAPT (final concentration $100 \mu \mathrm{mol} / \mathrm{l}$ ) or the equivalent amount of DMSO as a negative control. Total protein or RNA was harvested $48 \mathrm{~h}$ later. Protein and mRNA expression of VEGFR-1, VEGFR-2 and VEGFR-3 was measured by Western blotting and real-time PCR, respectively.

DAPT treatment and eNOS expression. For the assessment of the role of VEGFR-2 on eNOS regulation under DAPT treatment, $1 \times 10^{6} \mathrm{H} 5 \mathrm{~V}$ cells were cultured with DAPT $(100 \mu \mathrm{mol} / \mathrm{l})$, DAPT $(100 \mu \mathrm{mol} / \mathrm{l})+$ VEGFR-2 kinase inhibitor $(200 \mu \mathrm{mol} /$ $\mathrm{ml}$ ), or DMSO (equivalent amount) for $48 \mathrm{~h}$. To assess the effects of different concentrations of DAPT, $1 \times 10^{6} \mathrm{H} 5 \mathrm{~V}$ cells 
$\mathbf{A}$
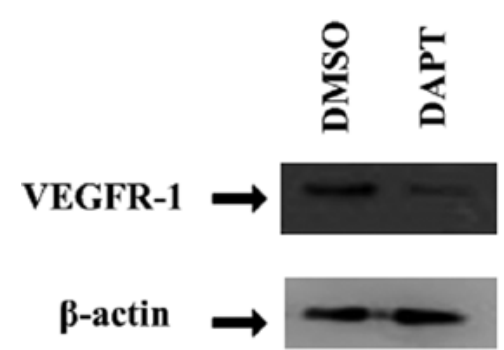

C

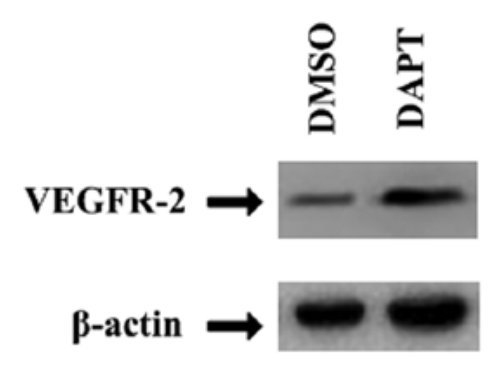

$\mathbf{E}$

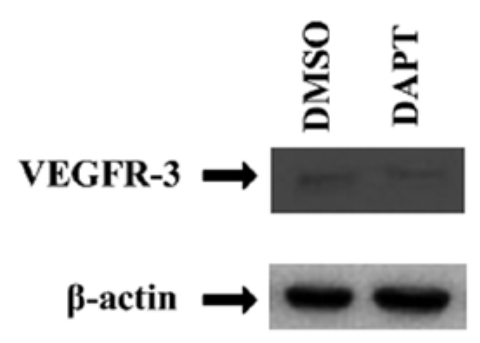

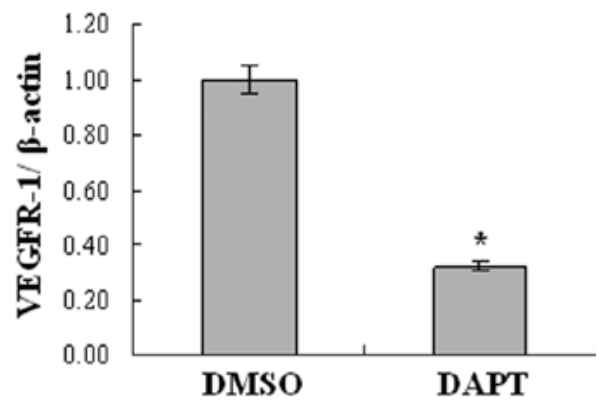

D

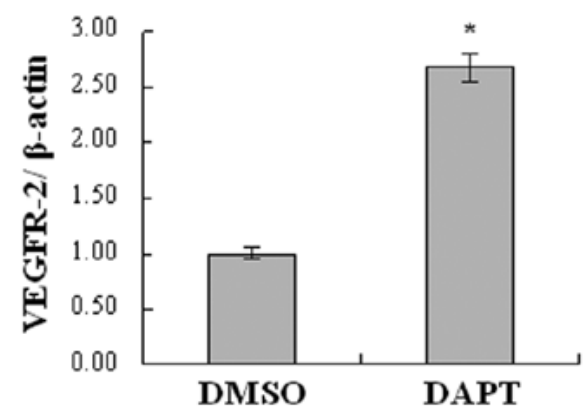

$\mathbf{F}$

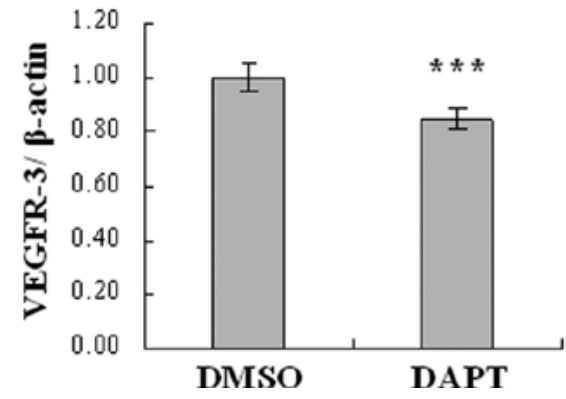

Figure 1. VEGFR expression level under DAPT treatment. (A) VEGFR-1 protein levels analyzed by Western blotting in $\mathrm{H} 5 \mathrm{~V}$ cells treated with $100 \mu$ mol/1 DAPT or DMSO after 48 h. $\beta$-actin was used as a loading control. (B) VEGFR-1 mRNA levels in H5V cells treated with $100 \mu$ mol/l DAPT after 48 h. Realtime PCR results were normalized using $\beta$-actin as a calibrator gene and are shown as average expression fold change relative to VEGFR-1 mRNA in the DMSO groups. (C) VEGFR-2 protein levels analyzed by Western blotting in H5V cells treated with $100 \mu \mathrm{mol} / 1$ DAPT or DMSO after $48 \mathrm{~h}$. $\beta$-actin was used as a loading control. (D) VEGFR-2 mRNA levels in H5V cells treated with $100 \mu \mathrm{mol} / 1$ DAPT after $48 \mathrm{~h}$. Real-time PCR results were normalized using $\beta$-actin as a calibrator gene and are shown as average expression fold change relative to VEGFR-2 mRNA in the DMSO groups. (E) VEGFR-3 protein levels analyzed by Western blotting in $\mathrm{H} 5 \mathrm{~V}$ cells treated with $100 \mu \mathrm{mol} / \mathrm{l}$ DAPT or DMSO after $48 \mathrm{~h}$. $\beta$-actin was used as a loading control. (F) VEGFR-3 mRNA levels in H5V cells treated with $100 \mu \mathrm{mol} / 1$ DAPT after $48 \mathrm{~h}$. Real-time PCR results were normalized using $\beta$-actin as a calibrator gene and are shown as average expression fold change relative to VEGFR-3 mRNA in the DMSO groups. Data are the means $\pm \mathrm{SD}$. ${ }^{*} \mathrm{P}<0.01,{ }^{* * *} \mathrm{P}>0.05$ vs. DMSO groups, $\mathrm{n}=3$ for each group.

were cultured with DAPT $(25,50,75$ and $100 \mu \mathrm{mol} / \mathrm{l})$ for $48 \mathrm{~h}$, and the equivalent amount of DMSO was used as a negative control. To assess the effect of different treatment times, $1 \times 10^{6}$ H5V cells were cultured with $100 \mu \mathrm{mol} / \mathrm{l}$ DAPT or DMSO (equivalent amount) for $0,6,12,24$ and $48 \mathrm{~h}$. Protein and mRNA expression of eNOS was measured by Western blotting and real-time PCR, respectively.

Western blotting. $\mathrm{H} 5 \mathrm{~V}$ cells were dissolved and boiled in Laemmli buffer for $10 \mathrm{~min}$. The samples were then separated by SDS-PAGE using $10 \%$ gel and transferred to polyvinylidene fluoride membranes (Bio-Rad) at $80 \mathrm{~mA}$ for $2 \mathrm{~h}$. After blocking with Tris (hydroxymethyl) aminomethane-buffered solution containing Tween-20 (TBST), the membranes were incubated with the primary antibody in TBST at $37^{\circ} \mathrm{C}$ for $2 \mathrm{~h}$. Secondary antibodies in TBST were added and incubated for $2 \mathrm{~h}$ at room temperature. Bound secondary antibodies were detected using an ECL detection system (Amersham Pharmacia Biotech). $\beta$-actin (Santa Cruz) was used as a loading control. The primary antibodies used were as follows: monoclonal VEGFR-1 antibody (Santa Cruz), polyclonal VEGFR-2 antibody (Cell Signaling), monoclonal VEGFR-3 antibody (eBioscience) and monoclonal eNOS antibody (Abcam).

RNA extraction and real-time PCR analysis. Total RNA was extracted from H5V cells using the RNeasy Mini kit (Qiagen, Valencia, CA, USA), and $1 \mu \mathrm{g}$ of total RNA was converted to cDNA by using the ExScript RT reagent kit (Takara, Otsu Shiga, Japan). Real-time PCR was carried out using the SYBR Green PCR Master Mix (ABI) and i-Cycler (Bio-Rad) according to the manufacturer's instructions. Target gene expression levels in each sample were subsequently normalized by the mRNA level of $\beta$-actin mRNA in the same mRNA 
A

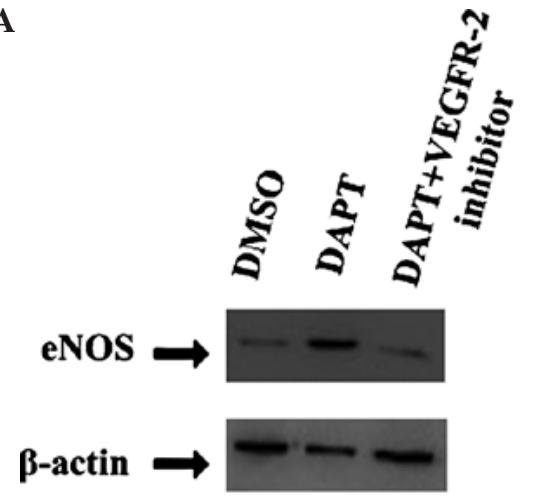

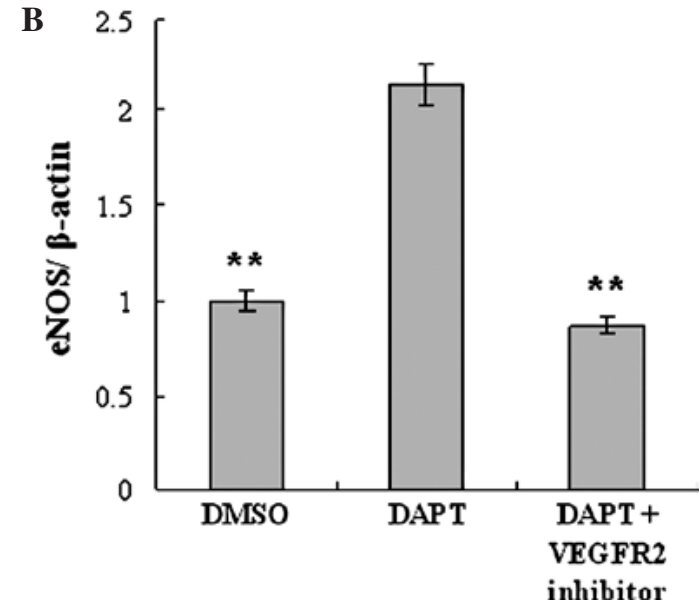

Figure 2. DAPT induces up-regulation of eNOS via VEGFR-2. (A) eNOS protein levels analyzed by Western blotting in H5V cells treated with DMSO, DAPT $(100 \mu \mathrm{mol} / \mathrm{l})$, or DAPT $(100 \mu \mathrm{mol} / \mathrm{l})+$ VEGFR-2 kinase inhibitor $(200 \mu \mathrm{mol} / \mathrm{ml})$ after $48 \mathrm{~h}$. $\beta$-actin was used as a loading control. (B) DAPT induced an increase in eNOS mRNA expression in H5V cells, and this effect was inhibited by VEGFR-2 kinase inhibitor. Real-time PCR results were normalized using $\beta$-actin as a calibrator gene and are shown as average expression fold change relative to eNOS mRNA in the DMSO groups. Data are the means \pm SD. ${ }^{* *} \mathrm{P}<0.05$ vs. DAPT groups, $\mathrm{n}=3$ for each group.

sample. The expression levels of each gene were expressed as normalized fold expression. Results were reported as the means \pm SD of triplicate experiments from three independent samples per group. Primers and probes included: VEGFR-1 forward primer 5'-ggcccgggatatttataagaac-3'; VEGFR-1 reverse primer 5'-ccatccattttaggggaagtc-3'; VEGFR-2 forward primer 5'-cagtggtactggcagctagaag-3'; VEGFR-2 reverse primer 5'-aca agcatacgggcttgttt-3'; VEGFR-3 forward primer 5'-gaatgag agccccggaac-3'; VEGFR-3 reverse primer 5'-ggtctccagaccagc aactc-3'; eNOS forward primer 5'-ccagtgccetgcttcatc-3'; eNOS reverse primer 5'-gcagggcaagttaggatcag-3'.

Statistical analysis. For all experiments triplicate measurements were obtained, and the results are shown as the means \pm SD. Statistical analysis of data was performed using the two-tailed Student's t-test. $\mathrm{P}<0.01$ and $\mathrm{P}<0.05$ were considered statistically significant.

\section{Results}

DAPT down-regulates VEGFR-1 expression. To assess whether the $\gamma$-secretase inhibitor DAPT affects VEGFR-1 expression, H5V cells were cultured with $100 \mu \mathrm{mol} / \mathrm{l}$ DAPT, or the equivalent amount of DMSO as a negative control. Western blot analysis revealed that the level of VEGFR-1 protein expression was decreased after $48 \mathrm{~h}$. Upon real-time PCR analysis, the mRNA level of VEGFR-1 was found to be down-regulated to $32.2 \%$ compared to the DMSO-treated H5V cells (Fig. 1A and B).

DAPT up-regulates VEGFR-2 expression. VEGFR-2 is the primary VEGF signaling receptor, and its expression is absolutely essential for vascular development and angiogenesis (6). Therefore, an in vitro model was used to determine VEGFR-2 expression under $100 \mu \mathrm{mol} / \mathrm{l}$ DAPT treatment. After $48 \mathrm{~h}$, VEGFR-2 protein expression was increased, as revealed by Western blotting, and the mRNA level was up-regulated by 2.68-fold compared to the control groups (Fig. 1C and D).
DAPT has no significant effect on VEGFR-3 expression. Although previous research showed that Notch signaling, the target of the $\gamma$-secretase inhibitor, altered VEGF responsiveness in murine endothelial cells by direct regulation of VEGFR-3 expression (7), our result failed to show any significant difference between the DAPT and DMSO groups in VEGFR-3 protein and mRNA expression (Fig. 1E and F).

DAPT induces up-regulation of eNOS via VEGFR-2. The eNOS expression level of H5V cells treated with DAPT was measured by Western blotting and real-time PCR, and the effect of VEGFR-2 was evaluated. After $48 \mathrm{~h}$ of DAPT treatment, eNOS expression was up-regulated at both the protein and mRNA level. Upon real-time PCR analysis, the level of mRNA expression of eNOS in the DAPT group was found to be increased by 2.13 -fold compared to the control. This effect was neutralized when $\mathrm{H} 5 \mathrm{~V}$ cells were pre-treated with the VEGFR-2 kinase inhibitor (Fig. 2).

DAPT up-regulates eNOS in a concentration-dependent manner. The eNOS protein level of the $75 \mu \mathrm{mol} / 1$ DAPT group was higher than that of the control, 25 and $50 \mu \mathrm{mol} / 1$ DAPT groups, but demonstrated no significant different with the $100 \mu \mathrm{mol} / \mathrm{l}$ DAPT group. The mRNA level of eNOS was up-regulated by DAPT in a concentration-dependent manner, which increased by 1.73 -fold in $\mathrm{H} 5 \mathrm{~V}$ cells treated with $25 \mu \mathrm{mol} / 1 \mathrm{DAPT}$, by 2 -fold in $\mathrm{H} 5 \mathrm{~V}$ cells treated with $50 \mu \mathrm{mol} / 1 \mathrm{DAPT}$, by 2.37 -fold in $\mathrm{H} 5 \mathrm{~V}$ cells treated with $75 \mu \mathrm{mol} / 1 \mathrm{DAPT}$ and by 2.24 -fold in $\mathrm{H} 5 \mathrm{~V}$ cells treated with $100 \mu \mathrm{mol} / 1 \mathrm{DAPT}$ compared to the expression level of DMSO-treated H5V cells (Fig. 3).

DAPT up-regulates eNOS in a time-dependent manner. Up-regulation of eNOS induced by DAPT treatment was found to be time-dependent. After treatment with $100 \mu \mathrm{mol} / 1$ of DAPT, the level of eNOS protein began to increase at $6 \mathrm{~h}$ and achieved peak levels at 12 and $48 \mathrm{~h}$, separately. As determined by real-time PCR analysis, mRNA expression 

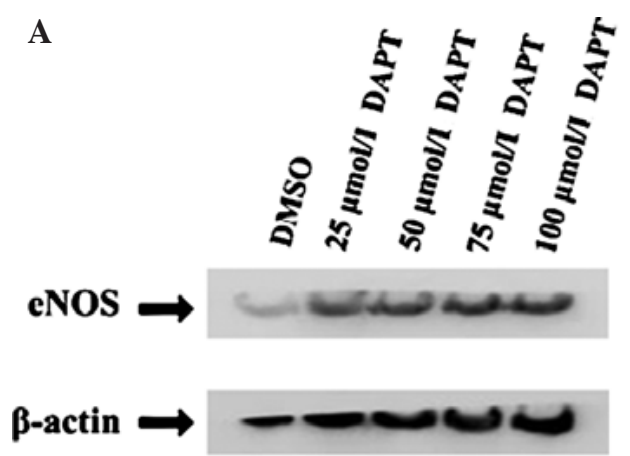

B

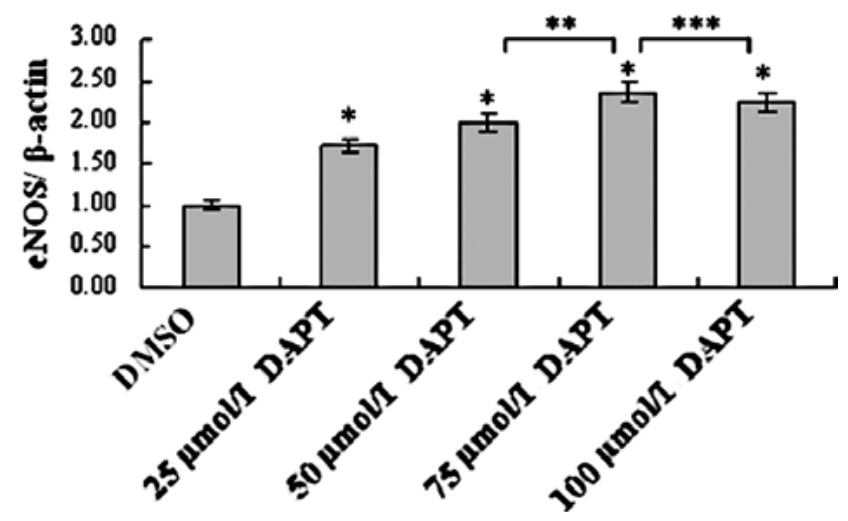

Figure 3. eNOS levels at different concentrations of DAPT. (A) eNOS protein levels analyzed by Western blotting in H5V cells treated with different concentrations of DAPT $(25,50,75$ and $100 \mu \mathrm{mol} / \mathrm{l})$ after $48 \mathrm{~h}$. An equivalent amount of DMSO was used as a negative control. $\beta$-actin was used as a loading control. (B) eNOS mRNA expression induced by different concentrations of DAPT after $48 \mathrm{~h}$. An equivalent amount of DMSO was used as a negative control. Real-time PCR results were normalized using $\beta$-actin as a calibrator gene and are shown as average expression fold change relative to eNOS mRNA in the DMSO groups. Data are the means $\pm \mathrm{SD}$. ${ }^{*} \mathrm{P}<0.01$ vs. DMSO groups, ${ }^{* *} \mathrm{P}<0.05,{ }^{* * * *} \mathrm{P}>0.05$ vs. $75 \mu$ mol/1 DAPT, $\mathrm{n}=3$ for each group.
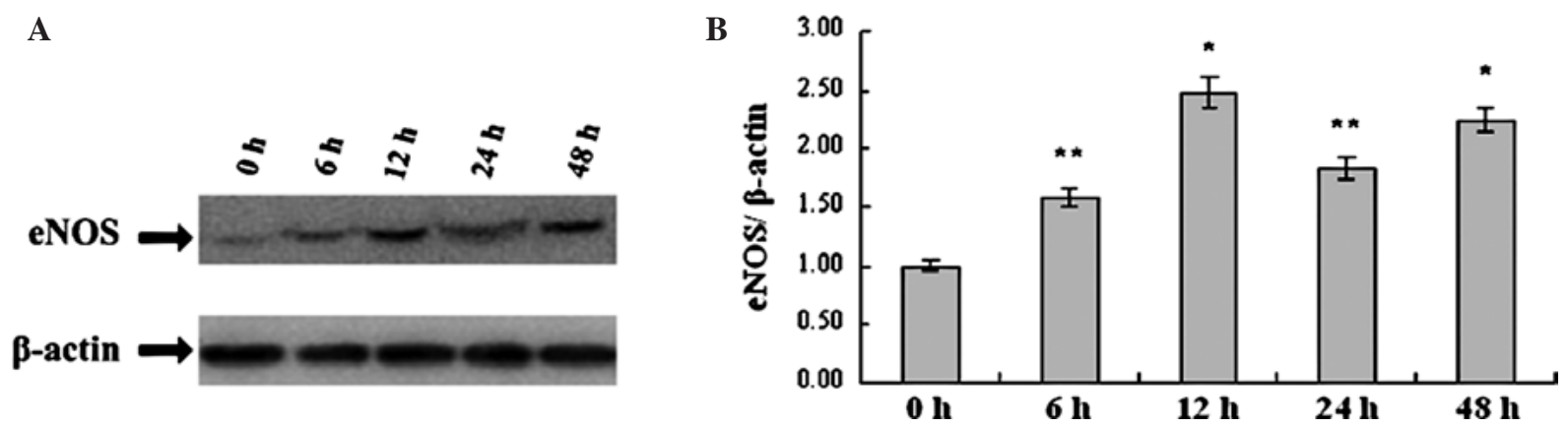

Figure 4. eNOS levels during the time course of DAPT treatment. (A) eNOS protein levels analyzed by Western blotting during the time course of $100 \mu$ mol/1 DAPT treatment. $\beta$-actin was used as a loading control. (B) eNOS mRNA expression in time course of $100 \mu$ mol/l DAPT treatment. Real-time PCR results were normalized using $\beta$-actin as a calibrator gene and are shown as average expression fold change relative to eNOS mRNA in the $0 \mathrm{~h}$ groups. Data are the means \pm SD. ${ }^{*} \mathrm{P}<0.01,{ }^{* *} \mathrm{P}<0.05$ vs. $0 \mathrm{~h}, \mathrm{n}=3$ for each group.

increased by 1.58 -fold at $6 \mathrm{~h}$, by 2.48 -fold at $12 \mathrm{~h}$ and by 2.25 fold at $48 \mathrm{~h}$, compared to the eNOS mRNA level of the control $\mathrm{H} 5 \mathrm{~V}$ cells at $0 \mathrm{~h}$. The alternating pattern of eNOS mRNA was similar to the pattern displayed by the eNOS protein as showed in the Western blot analysis (Fig. 4).

\section{Discussion}

Previous studies have shown that $\gamma$-secretase inhibitors may find application as pharmacological regulators of angiogenesis. In a T-cell acute lymphoblastic leukemia xenograft model, $\gamma$-secretase inhibitor indirectly suppressed tumor growth via disturbance of tumor angiogenesis (1). In another woundhealing model, bone marrow-derived vascular precursor cells pre-treated with the $\gamma$-secretase inhibitor DAPT failed to induce angiogenesis at the wound site in vivo (2). However, the mechanism of how $\gamma$-secretase inhibitors regulate angiogenesis has not been clearly understood. Given the important role of VEGFR and eNOS in angiogenesis, in vitro models were used to define the role of $\gamma$-secretase inhibitor DAPT on VEGFR and eNOS regulation.

In our study, after DAPT treatment for $48 \mathrm{~h}$, the expression level of VEGFR-1 was lower compared to the control groups, and VEGFR-2 was up-regulated compared to the control groups at both the protein and mRNA level. However, VEGFR-3 expression displayed no significant difference between DAPT-treated H5V cells and the control. To our knowledge, this is the first study to demonstrate that the $\gamma$-secretase inhibitor DAPT regulates VEGF signaling via alteration of the expression of VEGFR-1 and VEGFR-2. The mechanism will be investigated in subsequent research, and the results may aid in future $\gamma$-secretase inhibitor therapy strategies.

The effects of the $\gamma$-secretase inhibitor DAPT on eNOS expression were investigated. VEGF-A has been described to increase eNOS mRNA and protein expression in endothelial cells via VEGFR-2, but not VEGFR-1 in vivo, and the phosphatidylinositol 3-kinase (PI3-K) signaling pathway plays a crucial role in this process $(8,9)$. Blockage of VEGFR-2 via a monoclonal antibody was found to cause a marked reduction in the expression of eNOS and iNOS, which decreased NO generation and led to robust hypertension (10). Here, we found that the eNOS expression level was up-regulated in the DAPT-treated groups, at both the protein and mRNA level. This effect was neutralized by the VEGFR-2 kinase inhibitor. These findings indicate that DAPT regulates 
eNOS via VEGFR-2. Furthermore, our research showed that DAPT up-regulated eNOS in a concentration- and timedependent manner. Given that eNOS leads to vasodilation and better microvascular perfusion via NO production, it is rational to infer that enhancement of eNOS expression may offer some compensation in the microvascular after $\gamma$-secretase inhibitor therapy, which may be beneficial to normal tissues, but detrimental to cancer treatment. Further research by us will focus on this issue.

In summary, we initially demonstrated the relationship between VEGFR and eNOS under $\gamma$-secretase inhibitor treatment. The $\gamma$-secretase inhibitor down-regulated VEGFR-1, but up-regulated VEGFR-2, and the up-regulation of VEGFR-2 resulted in an increase in eNOS expression. Our findings suggest that administration of a $\gamma$-secretase inhibitor should be combined with disruption of eNOS or VEGF signaling to improve the anti-angiogenic therapeutic efficacy.

\section{Acknowledgements}

The authors would like to thank all members of our laboratory for the helpful discussions and comments on the manuscript. They also thank Dr Guo-kun Wang, of the Department of Cardiology, Changhai Hospital, Shanghai, China, and Dr Qiong Wang, of the Department of Medical Information, Changhai Hospital, Shanghai, China, for the technical assistance.

\section{References}

1. Masuda S, Kumano K, Suzuki T, et al: Dual antitumor mechanisms of Notch signaling inhibitor in a T-cell acute lymphoblastic leukemia xenograft model. Cancer Sci 100: 2444-2450, 2009.

2. Caiado F, Real C, Carvalho T and Dias S: Notch pathway modulation on bone marrow-derived vascular precursor cells regulates their angiogenic and wound healing potential. PLoS One 3: e3752, 2008.

3. Cook KM and Figg WD: Angiogenesis inhibitors: current strategies and future prospects. CA Cancer J Clin 60: 222-243, 2010.

4. Michel $\mathrm{T}$ and Vanhoutte PM: Cellular signaling and NO production. Pflugers Arch 459: 807-816, 2010.

5. Toda N, Ayajiki K and Okamura T: Interaction of endothelial nitric oxide and angiotensin in the circulation. Pharmacol Rev 59: 54-87, 2007.

6. Hirashima M: Regulation of endothelial cell differentiation and arterial specification by VEGF and Notch signaling. Anat Sci Int 84: 95-101, 2009.

7. Shawber CJ, Funahashi Y, Francisco E, et al: Notch alters VEGF responsiveness in human and murine endothelial cells by direct regulation of VEGFR-3 expression. J Clin Invest 117: 3369-3382, 2007.

8. Kroll $\mathrm{J}$ and Waltenberger J: VEGF-A induces expression of eNOS and iNOS in endothelial cells via VEGF receptor-2 (KDR). Biochem Biophys Res Commun 252: 743-746, 1998.

9. Wang Y, Nagase S and Koyama A: Stimulatory effect of IGF-I and VEGF on eNOS message, protein expression, eNOS phosphorylation and nitric oxide production in rat glomeruli, and the involvement of PI3-K signaling pathway. Nitric Oxide 10: 25-35, 2004.

10. Facemire CS, Nixon AB, Griffiths R, Hurwitz H and Coffman TM: Vascular endothelial growth factor receptor 2 controls blood pressure by regulating nitric oxide synthase expression. Hypertension 54: 652-658, 2009. 